\title{
Decision-making by nematodes in complex microfluidic mazes
}

\author{
Santosh Pandey*, Andrew Joseph, Roy Lycke, Archana Parashar
}

Department of Electrical and Computer Engineering, Iowa State University, Ames, USA.

Email: "pandey@iastate.edu

Received 5 September 2011; revised 9 October 2011; accepted 24 October 2011.

\begin{abstract}
Nematodes are microscopic, soil-dwelling worms that navigate through soil particles in search of food or a suitable host. Most nematode species employ a myriad of physical and chemical cues that define their navigation strategies. Here, we demonstrate a microfluidic method to observe and characterize the physical aspects of nematode navigation at real-time. The microfluidic devices comprise a series of interconnected T-maze or cylindrical structures of varying geometry. At each physical intersection, nematodes are given the choice to migrate left or right. We found that this decision-making of nematodes is influenced by the angle of intersection of T-maze structures. We further showed that nematodes can be passively directed to move in a linear direction by carefully adjusting the position and spacing of cylindrical obstacles in its path. The experiments were conducted on two nematodes (non-parasitic $C$. elegans and pigparasitic Oesophagostomum dentatum) and in the absence of any chemical or electrical stimulants.
\end{abstract}

Keywords: C. elegans; Navigation; Maze; Microorganism; Microfluidics

\section{INTRODUCTION}

Nematodes are the most ubiquitous, soil-dwelling microorganisms on Earth with over 20,000 known species. Particularly, the nematode Caenorhabditis elegans (C. elegans) is an important model organism for researchers in neuroscience, genetics, and behavioral sciences [1-4]. The primary reasons for choosing C. elegans as a small animal laboratory model organism are its microscopic size, transparent body, ease of culture, short lifespan, and a reasonable genome size [5-11]. Recent advances in vivo microfluidic testing $[12,13]$ have facilitated the monitoring of several behavioral facets of nematodes such as locomotion, growth, reproduction, and neuronal functioning [14-16]. In an interesting work by Qin and Wheeler [17], C. elegans were put in simple T-maze microfluidic channels and trained to produce a definite chemotactic response. Other micro-organisms (e.g. bacteria, fungi, and algae) have also been subjected to complex maze structures and their attraction/repulsion to chemical gradients or food reservoirs have been characterized.

Besides chemical cues, nematodes are presented with a wide range of physical interfaces in their microenvironment that need to effectively sensed and responded to. It may be difficult for an experimentalist to visually observe these physical interfaces or the navigation strategies employed by nematodes in the natural soil habitat. In this regard, microfluidics has proved to be a boon to experimental biologists. Microfluidics offers the unique possibility of creating two-dimensional microstructures, altering device dimensions in a controlled manner, and observing the interactions between microorganisms and artificial chip environment [18-22].

In this work, we demonstrate a microfluidic device with a range of interconnected T-shaped mazes and cylindrical structures to characterize nematode movement through a series of physical interfaces. Specifically, we designed four assays: 1) angled T-maze, 2) sequenced T-maze, 3) convex intersection maze, and 4) sequenced pillar array. The tests were conducted on non-parasitic $C$. elegans and pig-parasitic Oesophagotomum dentatum (O. dentatum). No chemical or electrical stimulants were used in our tests. The experimental results help us determine a probabilistic impact model based on the geometry of physical interfaces and the nematodes' interaction with hard surfaces. Based on our observations, it is reasonable to assume that, in the absence of any stimulants or repellents, a nematode navigates essentially blindly through an agarose gel medium with occasional halts and turns. Any interactions with physical interfaces in its path alter its direction of movement, depending on the angle of the physical interface with respect to its body line. As we will show in the case of sequenced pillar array, it is possible to engineer the location, angle, and interval of physical interfaces for nematodes in order to modify their natural non-linear direction of motion into a linear one. 


\section{MATERIALS AND METHODS}

\subsection{Microfluidic Fabrication}

The mask layouts were drawn in AutoCAD and sent to an outside vendor (Fineline Imaging, Colorado Springs, $\mathrm{CO})$ for printing the masks. The microfluidic devices were fabricated using standard soft lithography. Briefly, negative SU-8 photoresist (Microchem Corporation, Newton, MA) was cast onto a 3-inch silicon wafer by a single or double spin-coating technique (spin at 2800 r.p.m; soft bake at $95^{\circ} \mathrm{C}$ for 5 minutes; repeat) to achieve a $40 \mu \mathrm{m}$ or $80 \mu \mathrm{m}$ thick film. The SU-8 resist was exposed to near UV $(375 \mathrm{~nm})$ light at $10 \mathrm{~mJ} / \mathrm{cm}^{2}$ for 50 seconds and subsequently developed. A PDMS prepolymer (Sylgard 184 Silicone Elastomer Kit, Dow Corning Corporation, Midland, MI) was cast over the SU-8 old and cured on a hot plate at $70^{\circ} \mathrm{C}$ for 2 hours. The hardened PDMS structures were peeled off the SU-8 mold and fluid ports ( $2 \mathrm{~mm}$ diameter) were punched. Air plasma was exposed to the PDMS structures, which were then bonded to individual glass slides to create sealed microfluidic devices. A polyethylene tubing $(\mathrm{OD}=1 \mathrm{~mm}$, $\mathrm{ID}=0.58 \mathrm{~mm}$ ) was connected to a $1-\mathrm{mL}$ syringe and the devices were filled with $0.8 \%$ agarose $(\mathrm{w} / \mathrm{v})$. During the agarose-filling process, we ensured that the input ports and microchannels do not trap any air bubbles which would potentially block or disrupt a nematode's movement.

\subsection{Maintenance of Nematodes}

C. elegans were obtained from the Caenorhabditis Genetics Center (CGC) at University of Minnesota (St. Paul, USA). The C. elegans were cultivated at $25^{\circ} \mathrm{C}$ on Nematode Growth Medium (NGM) plates seeded with Escherichia coli OP50 bacteria. For our tests, L4 stage C. elegans were picked using a sterilized platinum wire. Levamisole-sensitive (SENS) and resistant (LEVR) $O$. dentatum were originally supplied by the Royal Veterinary and Agricultural School, Frederiksberg, Copenhagen and then reproduced at six to nine months interval by passage in pigs at Iowa State University. The L2 larvae isolates were maintained between passages in tap water refrigerated at $11^{\circ} \mathrm{C}$ (changed every 2 - 4 months).

\section{RESULTS AND DISCUSSION}

\subsection{Angled T-Maze Structures}

The first microfludic chip consists of six angled T-maze structures, each with a distinct angle of intersection.

A representative schematic of the T-maze is illustrated in Figure 1(a). Each T-maze structure consists of a 2 $\mathrm{mm} \times 300 \mu \mathrm{m} \times 80 \mu \mathrm{m}$ microchannel $(\mathrm{L} \times \mathrm{W} \times \mathrm{H})$ connected to one entry and two exit ports ( $2 \mathrm{~mm}$ diameter). The angles of intersection of the different T-maze structures chosen in our experiments are 15, 30, 45, 60, 75 or 90 degrees. The microchannels were filled with the molten agarose/buffer mixture. The agarose solution was allowed to cool ( $\sim 5$ minutes) to form a porous gel-like medium. In this study, both C. elegans (N2) and O. dentatum (SENS) were tested. The nematodes were dropped on the entry port and allowed to migrate into the microchannel. In the absence of any stimulant or deterrent, the nematodes moved freely in the microchannels. The nematodes' progression was observed at real-time and recorded using a Leica MZ16 stereozoom microscope coupled with a QICam 12-bit digital camera.

We were interested in observing the navigation strategies employed by nematodes at the angled intersection of the T-maze structure (Figure 1(a)). Specifically, we focused on characterizing a nematode's decision to turn left or right at the angled intersection of the T-maze. No reward was given on either side of the T-maze. A worm in the microchannel began by exploring its surrounding and eventually moved towards the angled intersection. Before reaching the intersection, the nematode was essentially unaware of the impeding physical interface and continued in its sinusoidal path (Figure 1(b)). Upon reaching the intersection, the nematode's head impacted the physical interface, then retracted and continued either to the right or left arm of T-maze. We found that the direction of movement after impact (i.e. left or right turn decision) was dependent on the angle of intersection $\left(\theta^{\circ}\right)$ of the T-maze and the angle at which the worms' head was incident upon the physical interface.

As shown in Figure 1(b), a nematode's head can reach the physical interface during the upstroke or downstroke of its sinusoidal movement. At $90^{\circ}$ angled interfaces, we observed that worms impacting on an upstroke tended to move to the left arm of the T-maze, while worms impacting on a downstroke tended to move to the right. A probabilistic impact model was derived to predict the outcome of nematode decision-making ability (i.e. left or right turn decision) during such physical intersections. In other words, by characterizing the probability that a certain worm would make a left or right turn upon impacting a physical interface in a certain position (Figure 2(a)), we could predict the direction of movement after a single or multiple physical interactions. At the $90^{\circ}$ angled interface, the phase of sinusoidal movement at the impact location (i.e. up- or downstroke impact) was relatively random within a large sample population $(n \geq 40)$. Hence, there was approximately $50 \%$ chance for a certain worm to turn left or right (Figure 2(b)). This observation is supported by Qin and Wheeler's work [5] where C. elegans showed no apparent preference to move towards the left or right outlet chamber of a $90^{\circ} \mathrm{T}$-maze in the absence of any reward. 


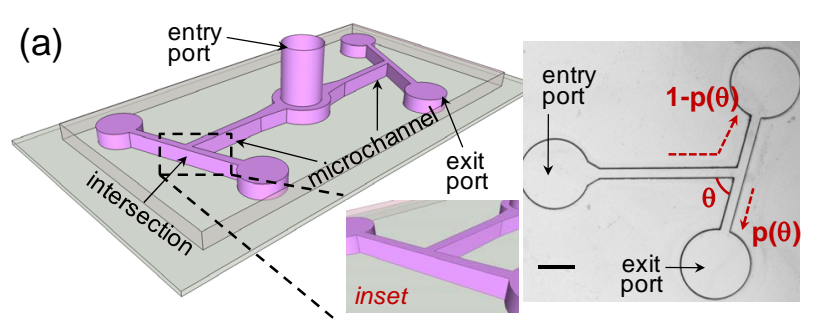

(b)

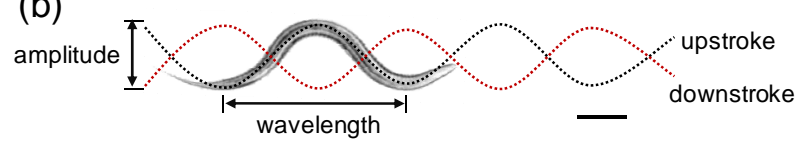

Figure 1. (a) Schematic of the microfluidic chip consisting of two angled T-maze structures. The angle of intersection is $\theta^{\circ}$ and the probability of right turn is $\mathrm{p}(\theta)$. Scale bar: $800 \mu \mathrm{m}$; (b) A nematode moves in a sinusoidal manner with a fixed amplitude and wavelength.
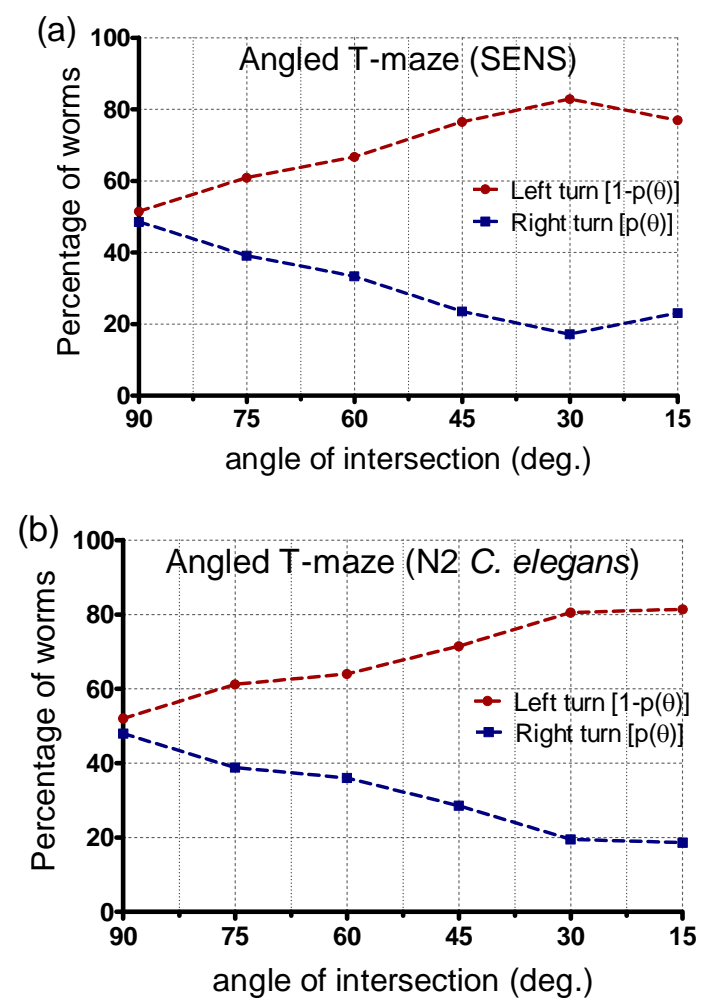

Figure 2. Experimental data from angled T-maze structures showing the percentage of worms making a right or left turn for a certain angle of intersection $\left(\theta^{\circ}\right)$. (a) Plot for SENS O. dendatum nematodes; (b) Plot for C. elegans.

In T-maze structures with angles of intersection other than $90^{\circ}$, we found that the probabilities for left and right turn altered significantly (Figure 2). As the angle of intersection $\left(\theta^{\circ}\right)$ was decreased from $90^{\circ}$, there was a decrease in the probability of turning right. In other words, as we reduced the angle of intersection from $90^{\circ}$ to $15^{\circ}$ (effectively, increasing the complementary angle from $90^{\circ}$ to $165^{\circ}$ ) in increments of $15^{\circ}$, we observed a linear decrease (or increase) in the probability of turning right (or turning left). In the next section, we show that the probability of right or left turn in a single T-maze can be used to predict worm locomotion in structures with multiple physical interfaces.

\subsection{Sequenced T-Maze Structures}

The second microfluidic chip consists of three distinct $\mathrm{T}$ maze structures in which multiple $(\sim 5-6)$ angled intersections are sequentially connected. Figure 3 shows a representative schematic of the microfluidic structure. Each maze comprises a microchannel (height $=80 \mu \mathrm{m}$; width $=300 \mu \mathrm{m}$; length $=600-4000 \mu \mathrm{m})$ connected with up to 5 exit ports (diameter $=2 \mathrm{~mm}$ ). Three sequenced T-maze structures were tested: 1) one consisting of 6 sequential $90^{\circ}$ angled intersections, 2) one consisting of 5 sequential $60^{\circ}$ angled intersections, and 3) one consisting of 5 decreasing angled intersections $\left(90^{\circ}, 75^{\circ}, 60^{\circ}\right.$, $45^{\circ}$ and $30^{\circ}$ ) (Figure 3 ).

The procedure for device filling, worm insertion, and imaging was similar to that in the previous angled Tmaze experiments. The microchannels were filled with a molten agarose/buffer mixture and subsequently allowed to cool down. A small population $(n=3-5, N>20)$ of nematodes were placed at the entry port. After a brief scouting time, the nematodes migrated into the microchannel and started exploring their surroundings. Thereafter, they reached the first physical interface and make a decision to turn right or left. If they turned left, they were collected in the first exit port. If they turned right, they

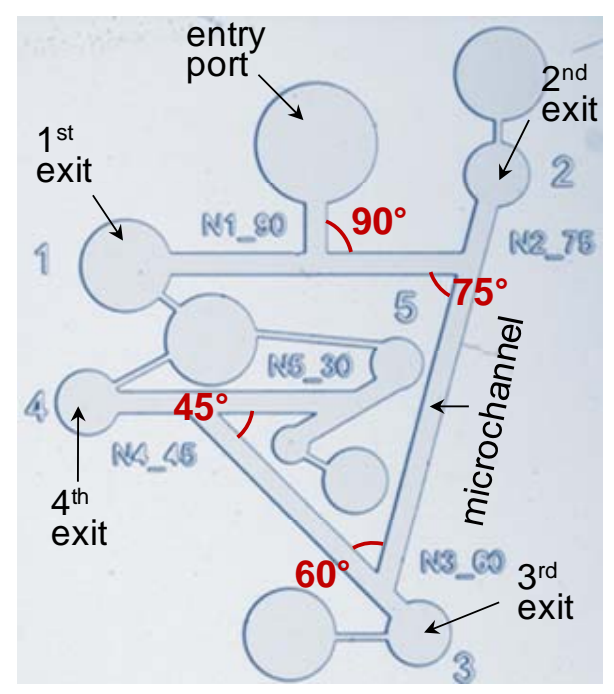

Figure 3. Snapshot of a fabricated sequenced T-maze structure showing the angles of intersection and exit ports at the first four physical interfaces. 
continued their movement before reaching the second interface. As before, a left turn at the second interface made them collect at the second exit port, while a right turn led them to the third interface. Thus, at each successive interface, a nematode was forced to make a decision on whether to turn right or left. At the end ofthe experiment, the number of worms in each exit port was counted. During chip design, the distance between any two consecutive interfaces was intentionally kept longer than six body wavelengths. Each experiment was conducted on a new chip and a fresh batch of worms.

Using the probabilistic impact model mentioned before, we estimated the probability that a given worm would end up in a certain exit port. We used the data from Figure 2 to estimate the bin probabilities (i.e. the probability a worm reaches a specific exit port or counting bin). For instance, consider the sequenced T-maze structure depicted in Figure 3. The model would suggest a $\sim 50 \%$ probability that a worm would be found in the first exit port, $\sim 31.5 \%$ probability that a worm would be found in the second exit port, $\sim 13 \%$ for the third exit port, $4.2 \%$ for the fourth exit port, and $\sim 0.7 \%$ for the fifth exit port. Figure 4 displays our experimental observations as compared to the theoretical probabilities (for both $C$. elegans (N2) and O. dentatum (SENS)). A total of $\sim 150$ worms were tested with $\sim 3-5$ worms in each experiment. All data sets were plotted using GraphPad Prism (GraphPad Software, La Jolla, CA); the percentage of worms found in each exit port are shown. In each sequenced Tmaze structure, the observed behavior of both nematode species closely matches the theoretical estimates generated by the impact model; no significant difference between the data sets was found ( $p$ value $>0.05)$.

\subsection{Convex Intersection Maze}

We next extended the angled T-maze structures to study the impact model at curved interfaces which may better resemble the worms' natural habitat. This microfludic chip comprises a single $90^{\circ} \mathrm{T}$-maze that has a convex, semi-spherical protrusion $(150 \mu \mathrm{m}$ in diameter) (Figure 5). Each maze consists of an entry port and two exit ports (2 mm diameter) connecting a $2 \mathrm{~mm} \times 300 \mu \mathrm{m} \times 80 \mu \mathrm{m}$ microchannel $(\mathrm{L} \times \mathrm{W} \times \mathrm{H})$.

The channels were filled with the agarose mixture and worms were inserted into the assay through the entry port. The worms were allowed to wander freely towards the convex protrusion where they made impact with the curved surface and decided to turn right or left. We defined four impact zones (L1, L2, R1 and R2; Figure 5(a))

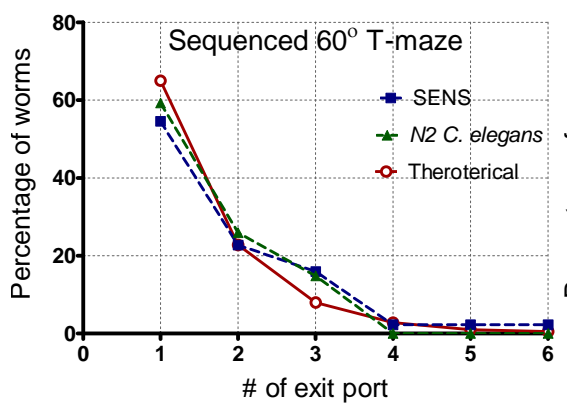

(a)

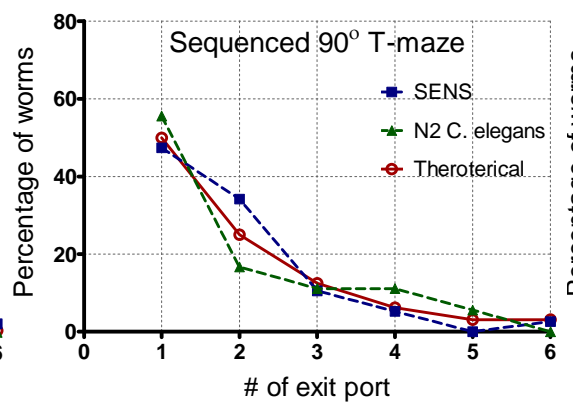

(b)

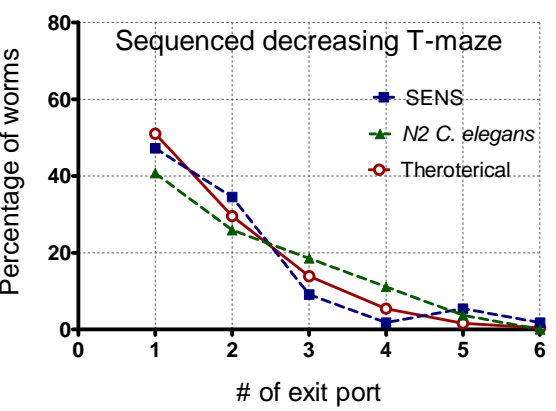

(c)

Figure 4. Experimental data from sequenced T-maze structures showing the percentage of worms collected at each exit port. (a) Plot for sequenced $60^{\circ}$ T-maze; (b) Plot for sequenced $90^{\circ}$ T-maze; and (c) Plot for sequenced decreasing T-maze.

(a)

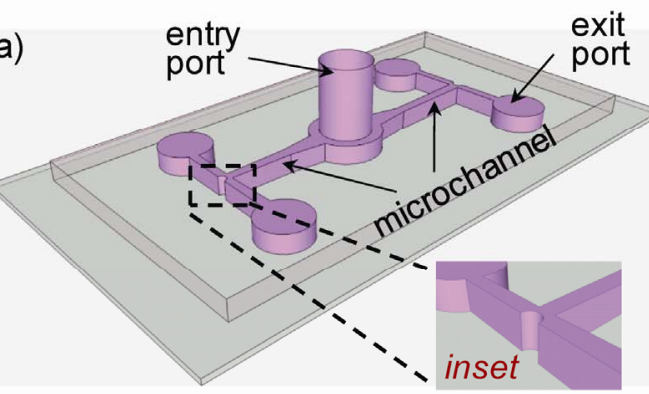

(b)

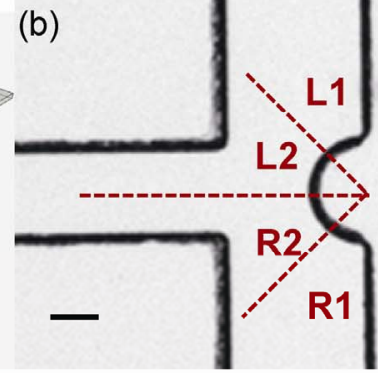

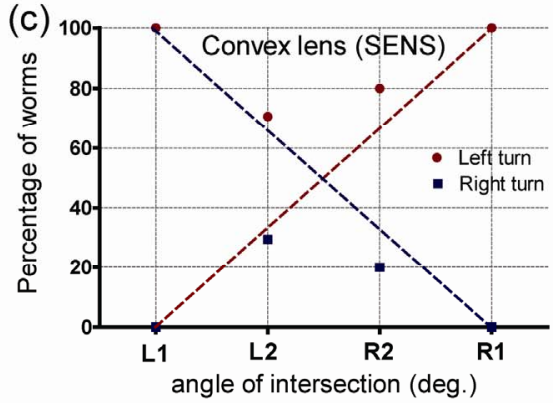

Figure 5. (a) Schematic of the microfluidic chip consisting of two convex intersection mazes; (b) Illustration of the convex structure showing the four zones of impact; and (c) Percentage of worm choosing a left or right turn upon making impact to either of the four impact zones. 
and characterized the decision-making ability (i.e. left or right turn) based on which zone of impact (Figure 5(b). In all our tests, we observed that nearly $100 \%$ of worms turned left (or right) after impacting the zone L1 (or R1). As the zone of impact changed from L1 towards R1 (or equivalently R1 towards L1) we observed a linear decrease in the probability of turning left (or equivalently of turning right). If the impact was exactly between zones L2 and R2 (i.e. a perpendicular impact), there is roughly a $50 \%$ probability of turning right or left.

\subsection{Sequenced Pillar Array}

Based on our results from convex intersection maze, we decided to test worm locomotion in an array of cylindrical structures. Previously, microfluidic chambers with three-dimensional pillar arrays have been used to mimic the natural soil microenvironment of nematodes and observe the adaptability in worm locomotion [16]. After several attempts, we fabricated an array of such pillar structures (each $300 \mu \mathrm{m}$ in diameter, $350 \mu \mathrm{m}$ center-tocenter distance) (Figure 6). Worms were inserted from an entry port and allowed to migrate towards the pillar array. With the above pillar dimensions, we observed that a nematode prefers to move in a linear direction. shown in the snapshots (taken every 1 second), a nematode efficiently uses the surface of a pillar structure to conform its body into a sinusoidal posture and continue towards the next pillar structure. This test was conducted on over $30 \mathrm{O}$. dendatum nematodes with good repeatability.

Lastly, we wished to revisit the mode of intersection between a nematode and a physical interface in the Tmaze structure. We analyzed all the videos of the single T-maze experiments for both $O$. dendatum and C. elegans. The data was collected from all angled T-maze structures and showed an interesting pattern of mode transitions for both nematode species under test. Figure 7(a) shows the modes of interaction for $O$. dendatum. Upon impact, most $O$. dendatum (green arrows) slide their head on the surface, slide off their body on the surface, and eventually leave the intersection. In some cases, they may attempt to probe the surface using their head by digging in, backing off their head, or backing off their entire body. Figure 7(b) shows the modes of interaction for C. elegans. Upon impact, most C. elegans (green arrows) slide their head on the surface, slide off their body on the surface, and eventually leave the intersection. However, compared to O. dendatum, there is an increased tendency to retract their entire body and leave the intersection.

\section{CONCLUSIONS}

We presented four microfluidic structures to study the interaction of nematodes with physical interfaces in the absence of any stimulants or repellents. An important observation was that the angle of intersection of the interface determined the decision-making ability of nematodes. A relationship between the angle of intersection and the probability of right or left turn was estimated from experiments using the T-maze structures. We found that this impact relation holds true even in complex T-maze structures with multiple interfaces. We also conducted similar tests using curved surfaces and constructed a pillar array to modify the natural, non-linear motion of nematodes. We found that nematodes use the pillar structures as support systems to guide their sinusoidal pattern of locomotion. By adequately arranging the position and size of the pillars, we demonstrated that the directionality of nematodes can be passively controlled. We hope that the presented method helps in the understanding of nematode locomotion, particularly the biomechanics of this fundamental worm behavior.
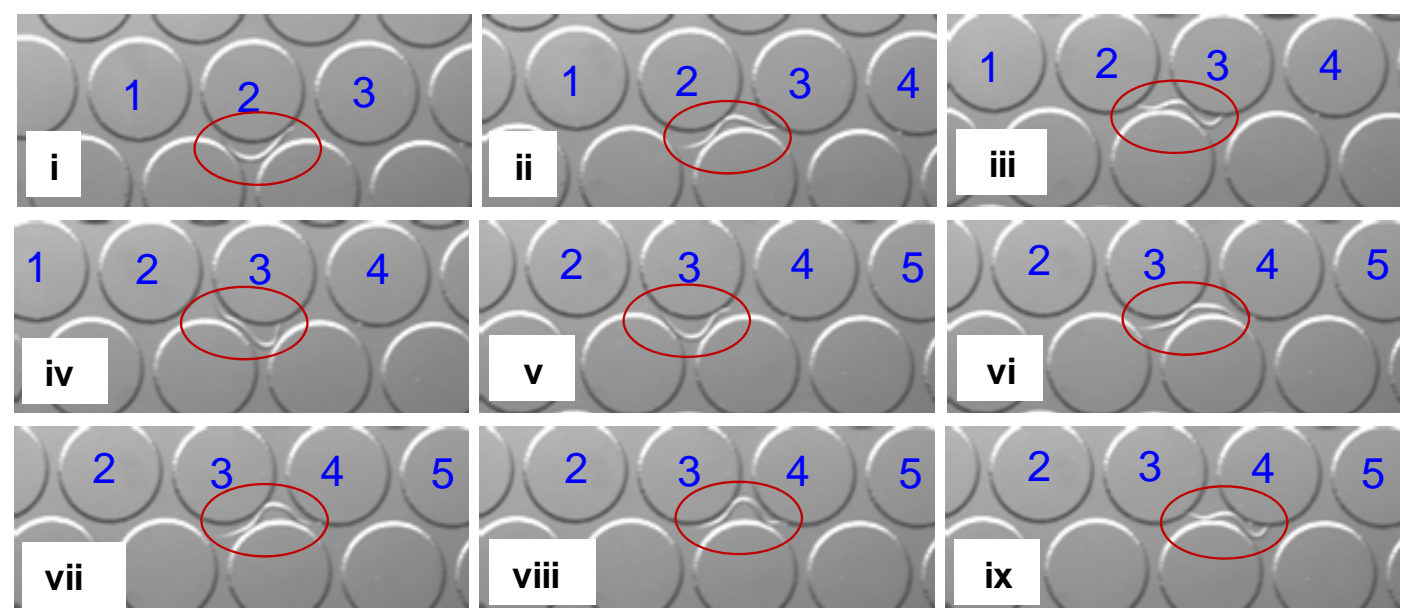

Figure 6. Snapshots of a single $O$. dendatum nematode (circled in red) traversing a series of pillars (numbered in blue). 

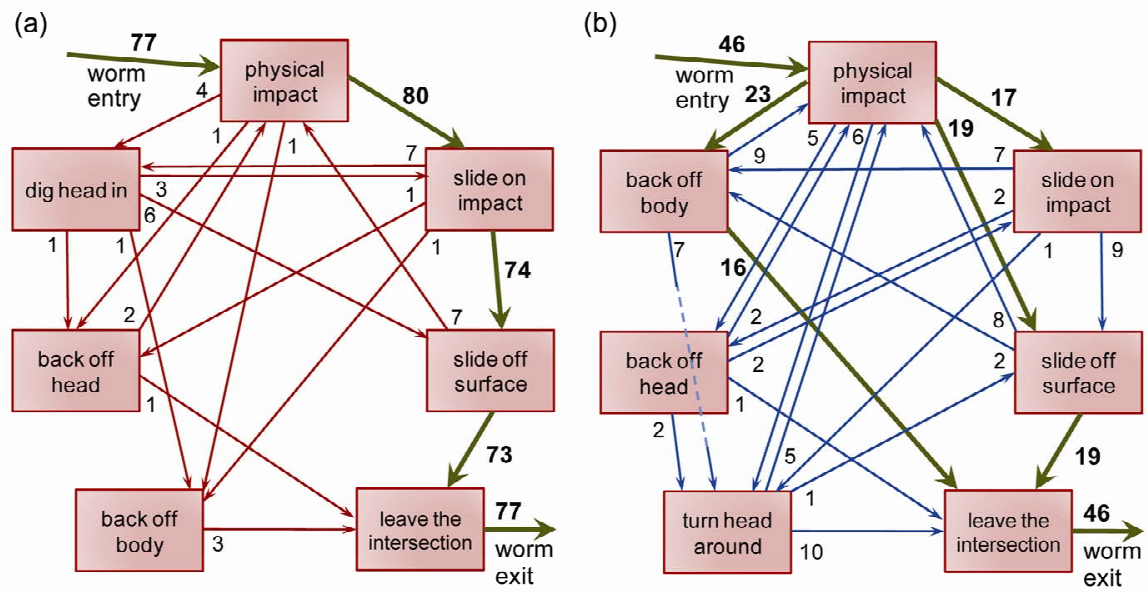

Figure 7. Modes of interaction for O. dendatum (a) and C. elegans (b) upon making impact with a single angled T-maze structure. The number of worms making each mode transition is marked next to the start of arrow. The green arrows denote the primary path in the circular graph.

\section{ACKNOWLEDGEMENTS}

We are thankful to R. J. Martin and A. P. Robertson for providing the nematodes. We are grateful to Chris Chu, Joseph Zambreno, and John Carr for their valuable advice and guidance on the experiments.

\section{REFERENCES}

[1] Rankin, C.H., Beck, C.D.O. and Chiba, C.M. (1990) Caenorhabditis elegans: A new model system for the study of learning and memory. Behavioural Brain Research, 37, 89-92. doi:10.1016/0166-4328(90)90074-O

[2] Wicks, S.R. and Rankin, C.H. (1997) The effects of tap withdrawal response habituation on other withdrawal behaviors: The localization of habituation in C. elegans. Behavioral Neuroscience, 111, 1-12. doi:10.1037/0735-7044.111.2.342

[3] D’Adamo, P., Wolfer, D.P., Kopp, C., Tobler, I., Toniolo, D. and Lipp, H.-P. (2004) Mice deficient for the synaptic vesicle protein Rab3a show impaired spatial reversal learning and increased explorative activity but none of the behavioral changes shwon by mice deficient for the Rab3a regulator Gdi1. European Journal of Neuroscience, 19, 1895-1905. doi:10.1111/j.1460-9568.2004.03270.x

[4] Wood, M.A., Kaplan, M.P., Park, A., Blanchard, E.J., Lombardi, T.L. and Abel, T. (2005) Transgenic mice expressing a truncated form of CREB-binding protein (CBP) exhibit deficits in hippocampal synaptic plasticity and memory storage. Learning \& Memory, 12, 111-119. doi: $10.1101 / 1 \mathrm{~m} .86605$

[5] Croll, N.A. (2009) Components and patterns in the behaviour of the nematode Caenorhabditis elegans. Journal of zoology, 176, 159.

doi:10.1111/j.1469-7998.1975.tb03191.x

[6] Croll, N.A. (1977) The location of parasites within their hosts: The behaviour of Nippostrongylus brasiliensis in the anaesthetised rat. International Journal for ParasitoLogy, 7, 195. doi:10.1016/0020-7519(77)90046-7

[7] Croll, N.A. (1977) Sensory mechanisms in nematodes.
Annual Review of Phytopathology, 15, 75.

doi:10.1146/annurev.py.15.090177.000451

[8] Niebur, E. and Erdos, P. (1991) Theory of the locomotion of nematodes-dynamics of undulatory progression on a surface. Biophysical Journal, 60, 1132-1146. doi:10.1016/S0006-3495(91)82149-X

[9] Niebur, E. and Erdos, P. (1993) Theory of the locomotion of nematodes: Control of the somatic motor neurons by interneurons. Mathematical Biosciences, 118, 51-82. doi:10.1016/0025-5564(93)90033-7

[10] Roussel, N., Morton, C.A., Finger, F.P. and Roysam, B. (2007) A computational model for C. elegans locomotory behavior: Application to multiworm tracking. IEEE Transactions on Biomedical Engineering, 54, 1786-1797. doi:10.1109/TBME.2007.894981

[11] Restif, C. and Metaxas, D. (2008) Tracking the swimming motions of C. elegans worms with applications in aging studies. International Conference on Medical Image Computing and Computer Assisted Intervention, 11, 35-42.

[12] Lockery, S.R., Lawton, K.J., Doll, J.C., Faumont S. and Coulthard, S.M. (2008) Artificial dirt: Microfluidic substrates for nematode neurobiology and behavior. Journal of Neurophysiology, 99, 3136-3143. doi:10.1152/jn.91327.2007

[13] Park, S., Hwang, H., Nam, S.W., Martinez, F., Austin, R.H. and Ryu, W.S. (2008) Enhanced Caenorhabditis elegans locomotion in a structured microfluidic environment. PLoS One, 3, e2550. doi:10.1371/journal.pone. 0002550

[14] Chronis, N. (2010) Worm chips: Microtools for C. elegans biology. Lab on a Chip, 10, 432-437. doi:10.1039/b919983g

[15] Crane, M.M., Chung, K., Stirman, J. and Lu, H. (2010) Microfluidics-enabled phenotyping, imaging, and screening of multicellular organisms. Lab on a Chip, 10, 15091517. doi: $10.1039 / \mathrm{b} 927258 \mathrm{e}$

[16] Rohde, C.B., Zeng, F., Gonzalez-Rubio, R., Angel, M. and Yanik, M.F. (2007) Microfluidic system for on-chip high-through-put whole-animal sorting and screening at 
subcellular resolution. Proceeding of the National Academy of Sciences of the United States of America, 104, 13891-13895. doi:10.1073/pnas.0706513104

[17] Qin, J. and Wheeler, A.R. (2007) Maze exploration and learning in C. elegans. Lab on a Chip, 7, 186-192. doi:10.1039/b613414a

[18] Petersen, M.B., Craven, J., Bjørn, H. and Nansen, P. (2000) Use of a migration assay for the separation of adult pyrantel-susceptible and -resistant Oesophagostomum dentatum. Veterinary Parasitology, 91, 141-145. doi:10.1016/S0304-4017(00)00264-8

[19] Kotze, A.C., Le Jambre, L.F. and O'Grady, J. (2006) A modified larval migration assay for detection of resistance to macrocyclic lactones in Haemonchus contortus, and drug screening with Trichostrongylidae parasites. Veterinary Parasitology, 137, 294-305. doi:10.1016/j.vetpar.2006.01.017
[20] Daugschies, A. and Ruttkowski, B. (1998) Modulation of migration of Oesophagostomum dentatum larvae by inhibitors and products of eicosanoid metabolism. International Journal for Parasitology, 28, 355-362. doi:10.1016/S0020-7519(97)00153-7

[21] Lorimer, S.D., Perry, N.B., Foster, L.M., Burgess, E.J., Douch, P.G.C., Hamilton, M.C., Donaghy, M.J. and McGregor, R.A. (1996) A Nematode Larval Motility Inhibition Assay for Screening Plant Extracts and Natural Products. Journal of Agricultural and Food Chemistry, 44, 2842-2845. doi:10.1021/jf9602176

[22] Wang, W., Shor, L.M., LeBoeuf, E.J., Wikswo, J.P., Taghon, G.L. and Kosson, D.S. (2008) Protozoan Migration in Bent Microfluidic Channels. Applied and Environ- mental Microbiology, 74, 1945-1949. doi:10.1128/AEM.01044-07 